\title{
Original
}

\section{Rapid Single Cell Detection of Lactic Acid Bacteria in the Beer Using Bioluminescence Method}

\author{
TOSHIHIRO TAKAHASH ${ }^{1 *}$, YASUKAZU NAKAKITA ${ }^{2}$, AND TAKESHI NAKAMURA ${ }^{1}$ \\ ${ }^{1}$ Group R\&D Strategy Department, SAPPORO HOLDINGS LTD., \\ 4-20-1 Ebisu, Shibuya-ku, Tokyo 150-8522, Japan \\ ${ }^{2}$ Frontier Laboratories for Value Creation, SAPPORO HOLDINGS LTD., \\ 10 Okatome, Yaizu, Shizuoka 425-0013, Japan
}

Received 29 March, 2018/Accepted 6 July, 2018

\begin{abstract}
In this study, we developed a system, known as MicroStar ${ }^{\mathrm{TM}}$ Rapid Microbe Detection System (RMDS), to detect Lactobacillus brevis, which usually requires 2-4 days for examination by the conventional plate count procedure, for beer quality control using a bioluminescence method within $24 \mathrm{hr}$ and also aimed to develop a technology to detect bacterial growth without the need for cultivation. We used a highly sensitive luminous reagent that increased the activity of the luciferin-luciferase reaction to $2.5 \times 10^{-18} \mathrm{~mol}$ ATP/0.2 $\mu$ land could detect even a single lactic acid bacterial cell. The limitation of the method was that ATP derived from the beer hindered bacterial measurement and the supply of energy source to secure ATP of lactic acid bacterial cell. The sample beer was filtered through a membrane filter, avoiding the formation of beer foam to the best extent, the filter was cleaned with $10 \%$ ethanol and $0.1 \%$ sodium hydrogen carbonate solution, and incubated on a GMY agar plate ( $1 \%$ glucose, $0.2 \%$ malic acid, $0.67 \%$ yeast nitrogen base, $1 \%$ agar; pH 5.2) at room temperature for $2 \mathrm{hr}$. Post incubation of the filter, bacterial cell count was measured with RMDS. This method could overcome the hindrance of ATP measurement and could stably detect lactic acid bacteria without the need for cultivation.
\end{abstract}

Key words : Rapid Detection / Lactobacillus brevis / ATP Bioluminescence.

\section{INTRODUCTION}

For beer quality control, generally, beer product samples extracted at a certain rate from the process are filtered through a membrane filter and the presence or absence of microbial colonies on the filter is confirmed by the agar plate method. Moreover, typically, 2-4 days are required to obtain the result using this method. Particularly, lactic acid bacteria, such as Lactobacillus spp., which proliferate even in beer with a bacteriostatic effect, need special care with respect to quality control in beer factory (Priest, 1995). We developed a system (known as RMDS) that detected lactic acid bacteria, which usually requires 2-4 days by standard microbial examination, for beer quality control within $24 \mathrm{hr}$ by combining the ATP method with a highly sensitive camera

*Corresponding author. Tel: +81-54-629-7980, Fax: +81-54629-3144 equipped with a photomultiplier tube and introduced it to a beer factory (Takahashi et al., 1999; Takahashi et al., 2000a). Next, we proposed and designed a system that could improve the sensitivity of the detection in RMDS and could detect microbial contamination without the need for cultivation.

There are some approaches for improving the detection sensitivity of RMDS. One method is increasing the activity of the luciferin-luciferase reagent via eliminating the ATP of microorganisms to enhance the emission reaction for detecting fewer cells (Japan Air Cleaning Association Symposium, 1997). The other method is that ATP expended by luminescence reaction is recycled from ADP using pyruvate orthophosphate dikinase that can be used for more sensitive detection because of the lengthy continuing luminescent reaction (Sakakibara et al., 2003). However, for detecting the microbial cells without cultivation in beer containing ATP, there are two limitations. First, intracellular ATP levels of 
the contaminants are extremely low, and second, luminescent compounds or materials with ATP derived from beer interfere the measurement. The factors described above lead to a low signal-to-noise ratio ( $\mathrm{S} / \mathrm{N}$ ratio) in RMDS. A layer of foam is formed at the interface of the beer when beer is poured into a cup-shaped filter funnel. When suction filtration is initiated in this state, the liquid portion of the beer is aspirated and differences are observed depending on the layer thickness and state of foam, but the foam remains on the membrane filter (Fig. 1). Even when the membrane filter is cleaned with sterile water, or with other similar solutions, the foam rises to the interface of sterile water, and water is aspirated earlier; thus, it is difficult to completely remove beer foam. After filtration of beer, presence of the gluelike substance that covers and clogs the surface of the filter is confirmed (Fig. 2). To decrease the luminescence derived from ATP of beer, it is necessary to remove this glue-like substance with the highest possibility from the filter. The detection limit concentration of ATP with light condensing time of $90 \mathrm{sec}$ and $120 \mathrm{sec}$ was $9.8 \times$ $10^{-18}$ and $4.9 \times 10^{-18} \mathrm{~mol} / 0.2 \mu \mathrm{l} \mathrm{spot}$, respectively, and

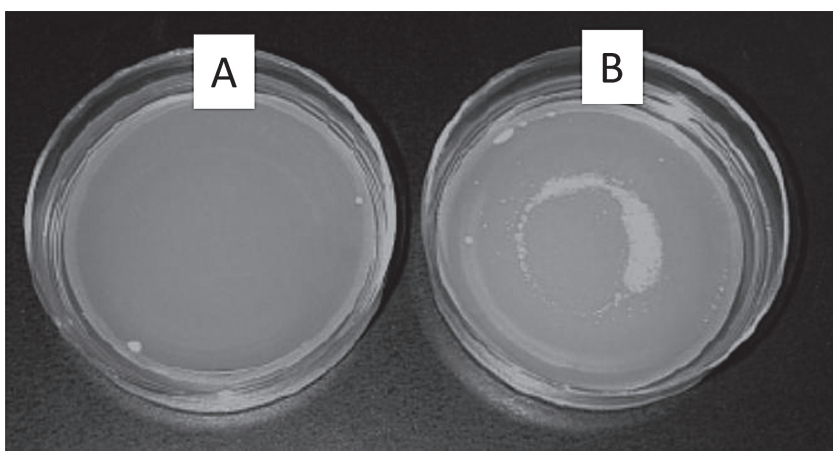

FIG. 1. Surface of the membrane filter after filtration of beer. Three hundred fifty milliliters of beer was filtrated with membrane filter. (A) After filtration the surface of membrane filter was washed with $10 \mathrm{ml}$ of $5 \%$ ethanol solution and the membrane filter was placed on M-NBB medium. ; (B) After filtration without surface washing. the sensitivity was 64-128-fold higher than that of the conventional system (Takahashi et al., 2000a). In case of $120 \mathrm{~s}$ of focusing, the spot was $2.5 \times 10^{-18} \mathrm{~mol} / 0.2$ $\mu \mathrm{l}$, which was 256 times higher than that by the conventional system. In theory, the current method exhibits sensitivity of detecting even a single lactic acid bacterial cell (Odaka et al., 1996). Furthermore, we confirmed that the detection sensitivity was almost similar to that of the method of ATP recycling by pyruvate, phosphate dikinase (PPDK) (Sakakibara et al., 2003). However, in this paper we have confirmed that when the filter containing bacterial cells was not incubated on a nutrient agar plate, microbial cell was hardly detectable. Therefore, to detect a single cell, treatment of the filter was necessary.

From the viewpoint of industrial use, we have resolved the cause of noise originating from beer ATP and have intensified the signal derived from ATP of lactobacilli as a detection target. Moreover, we could detect 1-100 lactic acid bacterial cells contaminating the beer.

\section{MATERIALS AND METHODS}

\section{Test strains}

L. brevis SBC 8001, L. brevis SBC 8002, and L. brevis SBC 8007 were obtained from Sapporo Brewing Culture Collection and were cultivated in MRS broth (Bacto lactobacilli MRS broth, Difco Laboratories, Detroit, USA) or commercial beer.

\section{Media and reagents}

M-NBB medium was composed of $0.5 \%$ casein peptone, $0.5 \%$ yeast extract, $0.2 \%$ meat extract, $0.05 \%$ Tween 80, 0.6\% $\mathrm{CH}_{3} \mathrm{COOK}, 0.05 \%$ L-malic acid, 0.2\% $\mathrm{K}_{2} \mathrm{HPO}_{4}, 0.02 \%$ L-cysteine- $\mathrm{HCl}, 1.5 \%$ glucose, and 1.5\% maltose; pH 5.2 (Nishikawa and Kohgo, 1996). ATP in the M-NBB medium was decomposed by a modification of the method of Sato and Nakakita (1995). TSA medium (1.5\% peptone, 0.5\% soy peptone, 0.5\% sodium chloride and 1\% agar; $\mathrm{pH}$ 7.5) of which ATP
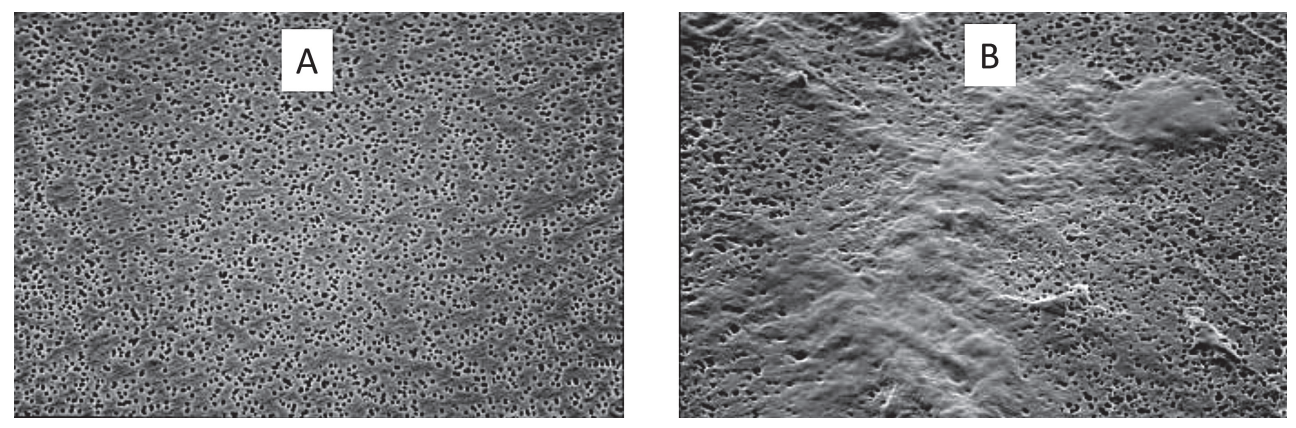

FIG. 2. Electron microscopic image $(\times 2,000)$ of Nuclepore filter, pore size $0.4 \mu \mathrm{m}$, surface. (A) Before filtration; (B) after filtration of beer $(633 \mathrm{ml})$. 
was decomposed by adenosine phosphate deaminase was purchased from Kikkoman (Noda, Japan). GMY medium contained $1 \%$ glucose, $0.2 \%$ malic acid, $0.67 \%$ yeast nitrogen base (YNB) (Difco, Detroit, USA), and $1 \%$ agar; $\mathrm{pH} 5.2$.

Composition of YNB, YNB w/o A.A (amino acid and ammonium sulfate), YNB w/o A. (amino acid) and
Bacto vitamin free yeast base (Difco, Detroit, USA) are shown in Table 1. Inorganic salt solution $(\times 10)$ contained 1,000 ppm potassium phosphate, 500 ppm magnesium sulfate, 100 ppm sodium chloride, and 100 ppm calcium chloride. Trace metal solution $(\times 10)$ contained 500 ppb boric acid, 40 ppb copper sulfate, 100 ppb potassium iodide, 200 ppb iron chloride, 400

TABLE 1. Compositions of YNB, YNB w/o A., YNB w/o A. A. and vitamin free in $\times 10$ solution were referred in Difco Manual (Difco, Detroit, USA)

\begin{tabular}{|c|c|c|c|c|}
\hline & $\begin{array}{l}\text { Bacto Yeast } \\
\text { Nitrogen Base }\end{array}$ & $\begin{array}{l}\text { Bacto Yeast } \\
\text { Nitrogen } \\
\text { Base w/o } \\
\text { Amino Acid }\end{array}$ & $\begin{array}{c}\text { Bacto Yeast } \\
\text { Nitrogen } \\
\text { Base w/o } \\
\text { Amino Acid } \\
\text { and Ammonium } \\
\text { Sulfate }\end{array}$ & $\begin{array}{l}\text { Bacto Vitamin } \\
\text { Free Yeast Base }\end{array}$ \\
\hline \multicolumn{5}{|l|}{$\mathrm{N}$ source } \\
\hline Ammonium sulfate & $0.5 \%$ & $0.5 \%$ & - & $0.5 \%$ \\
\hline \multicolumn{5}{|l|}{ C source } \\
\hline Bactodextrose & - & - & - & $1.0 \%$ \\
\hline \multicolumn{5}{|l|}{ Amino acid } \\
\hline L-histidine & 10ppm & - & - & 10ppm \\
\hline LD-methionine & 20ppm & - & - & 20ppm \\
\hline LD-tryptphan & 20ppm & - & - & 20ppm \\
\hline \multicolumn{5}{|l|}{ Vitamin } \\
\hline Biotin & $2 \mathrm{ppb}$ & $2 \mathrm{ppb}$ & $2 \mathrm{ppb}$ & - \\
\hline Calcium pantothenate & 400ppb & 400ppb & 400ppb & - \\
\hline Folic acid & $2 \mathrm{ppb}$ & $2 \mathrm{ppb}$ & $2 \mathrm{ppb}$ & - \\
\hline Inositol & $2 \mathrm{ppm}$ & $2 \mathrm{ppm}$ & $2 \mathrm{ppm}$ & - \\
\hline Niacin & 400ppb & 400ppb & 400ppb & - \\
\hline p-Aminobenzen acid & 200ppm & 200ppm & 200ppm & - \\
\hline Hydrogen chloride pyridoxine & 400ppm & 400ppm & 400ppm & - \\
\hline Riboflavin & 200ppb & 200ppb & $200 \mathrm{ppb}$ & - \\
\hline Hydrogen thiamine & 400ppb & 400ppb & 400ppb & - \\
\hline \multicolumn{5}{|l|}{ Trace element } \\
\hline Bolic acid & $500 \mathrm{ppb}$ & 500ppb & 500ppb & 500ppb \\
\hline Copper sulfate & 40ppb & 40ppb & 40ppb & 40ppb \\
\hline Potassium iodide & $100 \mathrm{ppb}$ & $100 \mathrm{ppb}$ & $100 \mathrm{ppb}$ & 100ppb \\
\hline Iron chloride & 200ppb & 200ppb & 200ppb & 200ppb \\
\hline Manganese sulfate & 400ppb & 400ppb & 400ppb & 400ppb \\
\hline Sodium molybdate & 200ppb & 200ppb & 200ppb & 200ppb \\
\hline Zinc sulfate & 400ppb & 400ppb & 400ppb & 400ppb \\
\hline \multicolumn{5}{|l|}{ Inorganic salt } \\
\hline Calcium phosphate monohydrate & $0.1 \%$ & $0.1 \%$ & $0.1 \%$ & $0.1 \%$ \\
\hline Magnesium sulfate & 500ppm & 500ppm & 500ppm & 500ppm \\
\hline Sodium chloride & 100ppm & 100ppm & 100ppm & 100ppm \\
\hline Calcium chloride & 100ppm & 100ppm & 100ppm & 100ppm \\
\hline
\end{tabular}


ppb manganese sulfate, 200 ppb sodium molybdate, and $400 \mathrm{ppb}$ zinc sulfate. All reagents were purchased from Wako Pure Chemical Industries (Osaka, Japan). $\mathrm{pH}$ of M-NBB medium and GMY medium was adjusted using $1 \mathrm{~N} \mathrm{HCl}$.

\section{Beer filtration}

A membrane filter (Isoporefilter with ring, pore size 0.4 um, Nihon Millipore Ltd. Tokyo, Japan) was attached to a funnel $(47 \mathrm{~mm}$ polysulfone holder, Advantec Ltd., Tokyo, Japan). After pouring the beer (350-633 ml) into the funnel, beer foam decreased, beer was allowed to stand until a part of the liquid level could be visually confirmed, and then suction filtration was performed. After completely filtering the beer, $20 \mathrm{ml}$ of $10 \%$ ethanol solution was used, and the membrane filter was aspirated and washed. Next, $100 \mathrm{ml}$ of $0.1 \%$ aqueous solution of sodium hydrogen carbonate was poured into a cup-shaped filter funnel for cleaning the surface of filter, maintained for $5 \mathrm{~min}$, and then suction filtration was performed. After complete aspiration, $100 \mathrm{ml}$ of $0.1 \%$ aqueous solution of sodium hydrogen carbonate was poured into the cup-shaped filter funnel, followed by suction filtration. After filtration, the membrane filter was incubated on GMY medium containing almost no ATP at room temperature for $2 \mathrm{hr}$.

\section{Electron microscopic observation}

The surface of membrane filter (Nuclepore filter, pore size $0.4 \mu \mathrm{m}$, Costar Scientific Co., Ltd., Cambridge, Mass., GBR) was platinum evaporated and then observed with electron microscope (HITACHI S-4000, Hitachi, Ltd., Tokyo, Japan).

\section{Conventional plate count (C.P.C.) method}

The cell culture solution was diluted with sterilized water to 10-50 cells $/ 100 \mu \mathrm{l}$, suspended in $100 \mathrm{ml}$ of sterilized water or $350 \mathrm{ml}$ of beer, and suction filtrated using a membrane filter of $\phi 47 \mathrm{~mm}$ (Cellulose mixed ester, black, pore size $0.45 \mu \mathrm{m}$, Advantec, Ltd.). After filtration, the filter was incubated on an MRS or M-NBB agar plate and cultured in an anaerobic box (EAN-140, Tabai Espec Corp., Osaka, Japan) at $30^{\circ} \mathrm{C}$ for 2-4 days. Then, the numbers of colonies on the filter were counted.

\section{RMD method}

The filters were processed using the RMD High Sensitivity Reagent Kit (Nihon Millipore). The moisture contained in the filter was dried at $35^{\circ} \mathrm{C}$ for 2 min, and $60 \mu \mathrm{l}$ of ATP extraction reagent was sprayed on the filter using an automatic reagent spray (Nihon Millipore). After drying at $35^{\circ} \mathrm{C}$ for $1.5 \mathrm{~min}, 60 \mu$ of luminescent reagent was sprayed by automatic spraying. This filter has the property of increasing transparency when containing moisture and becoming white when dried. Immediately, the filter was set on the emission detector of RMDS, and the generated photons were measured for 3.5-5 min. The performance and measurement conditions of the charge-coupled device (CCD) camera are as follows: quantum efficiency, $4.7 \%$; CCD noise value, 19; discriminator level, 26; bright spot size, 4.11; and discrimination value, 7 . Relative light unit was defined as the amount of photons accumulated for the measured time in the 300-pixel diameter circle. Cell number counting condition was set under this condition and measured by automatic counting.

\section{Measurement of the detection limit of ATP solution}

A standard ATP sheet was prepared as follows: ATP solution (100 mM solution; pH 7.5; Pharmacia, Upsala, Sweden) was diluted with sterilized water to obtain concentrations of $2.0 \times 10^{-17}, 9.8 \times 10^{-18}, 4.9 \times 10^{-18}$, $2.5 \times 10^{-18}$, and $1.2 \times 10^{-18} \mathrm{~mol} / 0.2 \mu \mathrm{l}$. The solution $(0.2 \mu \mathrm{l})$ was spotted on Isoporefilter and dried to fix ATP. A standard ATP sheet was used for RMDS as mentioned previously (Takahashi et al., 2000a).

\section{Measurement of cell number per colony}

The cell number per colony on the agar plate was measured using a fluorescence microscope (Micro Scanner, Daiichi Seimitsu Ltd., Yaizu, Shizuoka, Japan).

\section{Definition of viability}

Lactic acid bacteria were cultured in MRS liquid medium at $30^{\circ} \mathrm{C}$ for three days. After completion of the cultivation, the cells were centrifuged $(10,000 \times \mathrm{g}, 15$ min at $4^{\circ} \mathrm{C}$ ) and washed with saline. One point five milliliter of suspension of $10^{7}$ cells $/ \mathrm{ml}$ (number of cells was measured using a hemocytometer of Tomah) was prepared, and after centrifugation, cellular supernatant was discarded and $1.5 \mathrm{ml}$ of $70 \%$ ethanol was added to the precipitation and mixed. After holding at room temperature for $15 \mathrm{~min}$, the suspension was centrifuged, and the collected cells were suspended in physiological saline. A dead cell suspension was prepared following the same protocol and was mixed with an equal concentration of the bacterial suspension (100\% viable cells) immediately after cultivation.

\section{RESULTS AND DISCUSSION}

\section{Influence of foam derived from beer on luminescence}

The results of comparing the amount of luminescence when waiting for the foam to decrease and when suction filtration was performed immediately after pouring 350 $\mathrm{ml}$ of beer are shown in Fig. 3. When luminescence was measured after foam had decreased, the average light emission amount tended to decrease compared with 


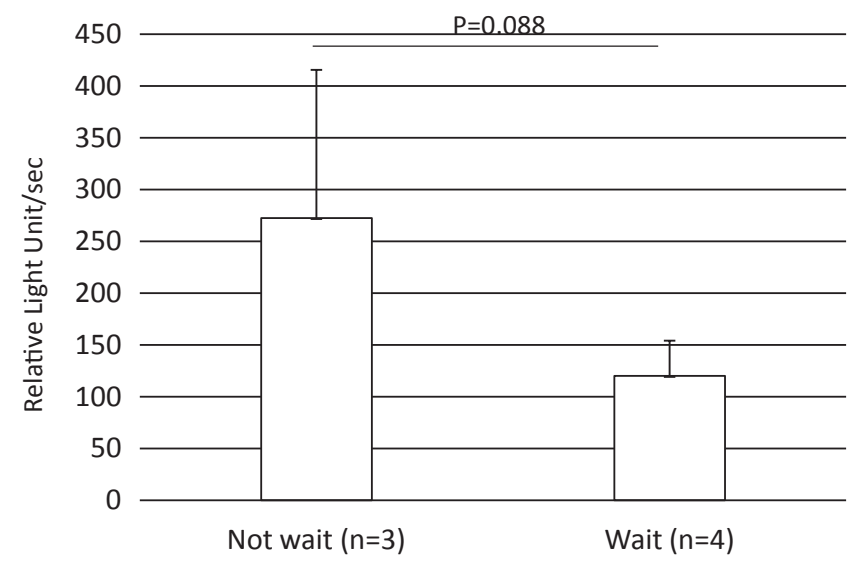

FIG. 3. Influence of beer residual foam on the amount of luminescence. After pouring the beer (350 ml) into the funnel, followed by filtration, the membrane filter was incubated on an agar plate containing 1\% glucose and measured by RMDS. "Not wait" indicates filtration without waiting for the foam to decrease, and "Wait" indicates filtration after waiting for the foam to decrease. The results are represented as mean $\pm S D$. Statistical analysis was performed using $\mathrm{T}$ test.

that observed when the luminescence was measured immediately after pouring $(P=0.088)$. Thus, the residual foam would increase the emission of ATP derived from beer.

When suction filtration was performed with foaming, it was difficult to remove components of the beer from the filter, and extracellular ATP increased the measurement value of RMDS. Hence, extra care should be taken while pouring the beer into the funnel to avoid foaming as much as possible, and after filtration of the beer, bubbles should be removed by rinsing the filter surface with low concentration ethanol solution.

\section{Effect of detergent for washing the filter}

An enzymatic method to decompose ATP contained in the beer is available (Sakakibara et al., 2003), but the detection of cells is influenced by using sufficient amount of enzyme for removing extracellular ATP derived from beer, which is extremely high than the ATP contained in bacterial cells. Therefore, it was assumed that an appropriate non-enzymatic reagent is better for washing and could serve as an inexpensive method for industrial use. The components remaining on the filter are primarily $\beta$-glucan, iso- $\alpha$ acid, and proteins; thus, alkali surfactants and high concentration of alcohol were expected to have a cleaning effect. However, these detergents are generally used for the extraction of ATP from the cells and there is a possibility of leak the intracellular ATP while cleaning the membrane filter; therefore, mild cleaning condition is required. It has been reported that sodium citrate enhances the cleaning

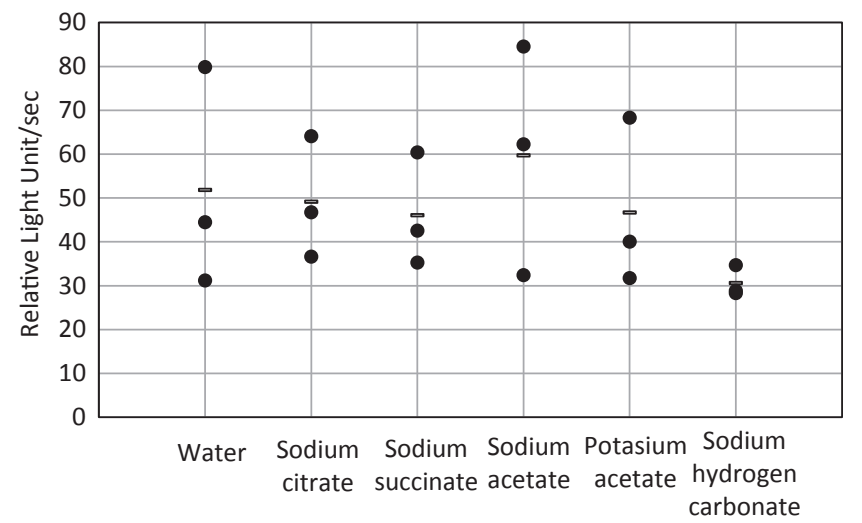

FIG. 4. Effect of detergent on decreasing the amount of luminescence. Beer (350 ml) was filtered as usual. One-hundred microliters of each detergent $(0.01 \%$ sodium citrate and others at $0.1 \%$ ) was poured into the funnel and retained for $5 \mathrm{~min}$. After suction, equal amount of the detergent was used for washing. Membrane filter was incubated on an agar plate containing 1\% glucose for 30 min at room temperature and measured by RMDS ( $n=3)$. Symbols; $\mathbf{O}$; measured value, $\mathbf{-}$ mean value.

effect by chelation (Ogino and Hayashi, 1977). Hence, we considered that chelation was effective for cleaning the membrane filter. The cleaning effects of an organic acid salt solution (containing sodium citrate, sodium succinate, potassium acetate, and sodium acetate) and weak alkaline solution (sodium hydrogen carbonate), which do not harm the microorganisms and can act as a detergent for washing the organic substances in the beer, were investigated (Fig. 4). Compared with distilled water, the organic acid salt solution did not exhibit a considerable cleaning effect. Beer components are not considered to be completely washed off if residual amount is observed on the membrane filter. Therefore, a treatment that could stably reduce extracellular ATP was required for measuring RMDS. For example, the condition wherein beer extracellular ATP is higher even once in 10 times leads to a false positive result. Thus, it was considered that the cleaning effect was not so high and that the results of cleaning with water and organic acid solution varied. However, the cleaning effect of sodium hydrogen carbonate was stable and showed better result than that of organic acid as shown in Fig. 4, although there was no statistical difference with Dunnett's test. Thus, sodium hydrogen carbonate can be used for cleaning the protein on the filter and exhibited low luminescence.

\section{Selection of basal medium without increasing the luminescence derived from component}

To further reduce the ATP remaining on the filter, we examined the condition to hold the filter on the agar 


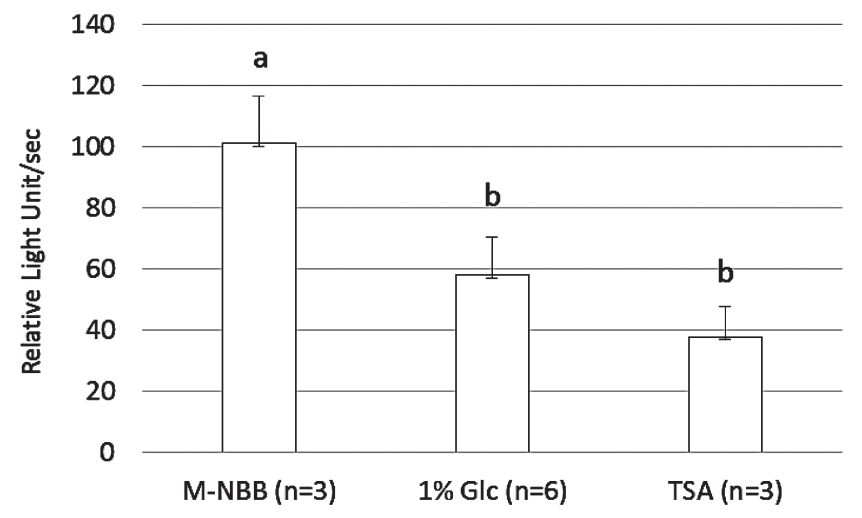

FIG. 5. Effect of the composition of agar plate on the amount of luminescence. Beer (350 ml) was filtered as usual. After filtration, the membrane filter was incubated on the agar plate containing M-NBB medium (M-NBB), 1\% glucose (Glc) or TSA medium (TSA) for 1-2 $\mathrm{h}$ and measured by RMDS. The results are represented as mean $\pm \mathrm{SD}$. Statistical analysis was performed using Tukey's test. The different letters indicate statistical differences $(P<0.01)$.

plate. Fig. 5 shows the relationship between each agar plate, containing various nutrients, incubated with the membrane filter and the amount of luminescence of the filter. In case of the membrane filter incubated in an agar plate containing M-NBB medium treated with apyrase (Sato and Nakakita, 1995), the amount of luminescence was higher than that of $1 \%$ glucose agar and TSA medium. By comparing 1\% glucose agar composed only of glucose and agar, TSA medium highly degraded ATP using adenosine phosphate deaminase and $\mathrm{M}-\mathrm{NBB}$ medium, much ATP remained in the M-NBB medium, and the difference in the amount of ATP contained in each medium was thought to influence the measurement. Hereafter, in this study, we examined the number of detected bacteria using 1\% glucose agar which did not use enzyme and could be prepared easily and inexpensively.

\section{Effect of nutrients on the number of detected cells}

To detect a single lactic acid bacterial cell, it was necessary to increase the amount of ATP above a certain level. Therefore, cells were incubated for a period of $2 \mathrm{hr}$ in an agar plate containing nutrients (sugar; organic acid; and inorganic salts) at a suitable $\mathrm{pH}$, leading to an increase in ATP. To determine the nutrients and conditions that could increase intracellular ATP, we examined agar plates containing various sugars or organic acids. Comparison of different sugar source using glucose, fructose, galactose, it appeared that the number of bacteria detected for glucose was somewhat larger, but it was not recognized as a clear difference (Table 2A). In test A using L. brevis SBC8007 cultivated in beer, there was no significant difference between the
TABLE 2. Effect of sugar in the agar plate on detecting cells.

\begin{tabular}{lccc}
\hline \multicolumn{1}{c}{ Experimental condition } & \multicolumn{2}{c}{ Count $(\mathrm{n}=2)$} & Average \\
\hline Base agar & 52 & 58 & 55.0 \\
Base agar $+1 \%$ Glucose & 73 & 62 & 67.5 \\
A Base agar $+1 \%$ Galactose & 47 & 64 & 55.5 \\
Base agar $+1 \%$ Fructose & 53 & 63 & 58.0 \\
Contactless & 1 & 0 & 0.5 \\
\hline Base agar & 11 & 1 & 6.0 \\
Base agar $+0.1 \%$ Glucose & 21 & 28 & 24.5 \\
Base agar $+0.5 \%$ Glucose & 21 & 19 & 20.0 \\
B Base agar $+1.0 \%$ Glucose & 28 & 22 & 25.0 \\
Base agar $+2.5 \%$ Glucose & 16 & 35 & 25.5 \\
Base agar $+5.0 \%$ Glucose & 21 & 21 & 21.0 \\
Base agar $+10.0 \%$ Glucose & 25 & 27 & 26.0 \\
\hline
\end{tabular}

(A) Cells suspended in sterilized water (L. brevis SBC 8007 cultivated in beer) were filtered, and the filter was incubated on agar plate and measured by RMDS $(n=2)$. "Contactless" indicates that measurement was performed without incubation on the agar plate. Glucose, galactose, or fructose (1\%) was added to the agar plate with $0.2 \%$ malic acid and $0.67 \%$ YNB ("Control"); pH 5.2. (B) Influence of glucose concentration in the agar plate on detecting cells. Cells suspended in sterilized water ( $L$. brevis SBC 8001 cultivated in MRS medium) were filtered and measured by RMDS $(n=2)$. Glucose was added to the agar plate containing $0.2 \%$ malic acid and $0.67 \%$ YNB; pH 5.2 .

addition and the absence of saccharides. When it was not brought into contact with nutrient agar, it was hardly detectable. Few differences were observed in the detection count when glucose concentration was in the range of $0.1-10.0 \%$ (Table $2 \mathrm{~B}$ ). In test $\mathrm{B}$ using $L$. brevis SBC 8001 cultivated in MRS medium, when glucose was not added, the detection count decreased considerably. Depending on the strain used for the test or preculture conditions, the demand for saccharides was considered to be different.

Moreover, the detection count tended to decrease in the agar plate without organic acid compared with that in the agar plate with malic acid or citric acid, and the decrease was particularly high in the agar plate with lactic acid (Table 3). Lactic acid was thought to have a negative effect on the metabolism of ATP.

Tables $4 \mathrm{~A}$ and $\mathrm{B}$ show whether substrates other than saccharides and organic acids are involved in enhancing the amount of luminescence. As shown in Fig. 4A, there were hardly any differences in the detection count by YNB w/o A. A., such as L-histidine, LD-methionine, and LD-tryptophan; YNB w/o A; and Vitamin Free, such as biotin, calcium pantothenate, folic acid, inositol, niacin, aminobenzene acid, hydrogen chloride pyridoxine, ribo- 
TABLE 3. Effect of organic acids in the agar plate on detecting cells.

\begin{tabular}{llrc}
\hline \multicolumn{1}{c}{ Experimental condition } & \multicolumn{2}{c}{ Count $(n=2)$} & Average \\
\hline Base agar & 53 & 61 & 57.0 \\
Base agar $+0.2 \%$ malic acid & 73 & 62 & 67.5 \\
Base agar $+0.2 \%$ citric acid & 64 & 71 & 67.5 \\
Base agar $+0.2 \%$ lactic acid & 39 & 36 & 37.5 \\
\hline
\end{tabular}

Cells suspended in sterilized water ( $L$. brevis SBC 8007) were filtered and measured by RMDS $(n=2)$. Organic acids $(0.2 \%)$ were added to the agar plate containing $1 \%$ glucose and $0.67 \%$ YNB; pH 5.2 ("Control").

TABLE 4. Effect of ammonium sulfate, amino acids, and vitamins in the agar plate on detecting cells.

\begin{tabular}{llccc}
\hline Experimental condition & \multicolumn{2}{c}{ Count $(\mathrm{n}=2)$} & Average \\
\hline Control (YNB) & 58 & 62 & 60.0 \\
A & YNB w/o A.A. & 61 & 60 & 60.5 \\
YNB w/o A. & 56 & 58 & 57.0 \\
& Vitamine free & 59 & 52 & 55.5 \\
\hline Control (YNB) & 30 & 27 & 28.5 \\
+salt solution, trace metal & 23 & 33 & 28.0 \\
B & +salt solution & 32 & 31 & 31.5 \\
+trace metal & 19 & 15 & 17.0 \\
- YNB & 23 & 17 & 20.0 \\
\hline
\end{tabular}

YNB (0.67\%), YNB w/o A. A. (0.17\%), YNB w/o A. (0.67\%), vitamin free $(1.67 \%)$, salt mixture $(0.67 \%)$ and/or trace metal mixture $(0.67 \%)$ was added to the agar plate containing $1 \%$ glucose and $0.2 \%$ malic acid; $\mathrm{pH}$ 5.2. The concentrations of YNB, YNB w/o A. A., YNB w/o A., vitamin free were referred to their catalog. The test strains in Tables $4 A$ and $4 B$ were $L$. brevis SBC 8007 and SBC 8002 cultivated in beer, respectively $(n=2)$.

flavin, and hydrogen thiamine, particularly from the components included in YNB. In case of addition of the inorganic salt mixture to the agar plate containing $1 \%$ glucose and $0.2 \%$ malic acid, the detection count was almost similar to that of YNB (Table 4B). However, addition of trace metal mixture decreased the detection count. Moreover, the detection count decreased without YNB. Adequate detection was observed by adding sugar, organic acid, and YNB; addition of trace metal instead of inorganic salt reduced the detection count. It has been reported that mineral requirements for lactic acid bacteria growth are complex (Boyaval, 1989). In our test strains, inorganic salt was required to produce ATP, but trace metal hardly contributed to detect the cells. Therefore, addition of glucose, malic acid, and inorganic salt was able to increase the amount of luminescence derived from the ATP of cells. Although the effective components contained in inorganic salt solution are not specified, it is speculated that phosphate is effective for producing ATP. Thus, it was confirmed that addition of $1 \%$ glucose, $0.2 \%$ organic acid (malic acid or citric acid), and YNB solution to the agar plate (GMY medium shown in MATERIALS AND METHODS) improved the detection count.

Furthermore, using the GMY agar plate, the influence of $\mathrm{pH}$ on the number of detected count of $L$. brevis SBC 8001 was investigated, and it was observed that the detection count was high in the acidic pH (Fig. 6A). In the test with L. brevis SBC 8007 cultivated in beer, the detection count was almost similar between $\mathrm{pH} 3.8$ and 7.0 and decreased at pH 9.0 (data not shown). The upper limit of optimum $\mathrm{pH}$ varies between strains or the physiological state of the cell, e.g., condition of the pre-culture or strain (Boyaval P., 1989). In the process controlled medium at the beer factory, the medium in the acidic range are often used for the microorganisms growing in the beer environment at the acidic $\mathrm{pH}$ (Hill A. E., 2015). Thus, it would be practical to adjust the range of $\mathrm{pH}$ between 3.8 and 5.5 .

The amount of time required for the substrate in the agar plate to be absorbed by the cells to increase ATP was investigated, and the relationship between the duration of incubation of the membrane filter on the agar plate and detection count was determined (Fig. $6 \mathrm{~B}$ ). A retention time is required for the cells to absorb nutrients from the agar plate and produce ATP. At 7 min of incubating the filter on the agar plate, cells were hardly detected. The detection count of cells was less at 30 min post incubation. A time period of more than 2 hr post incubation was stable for detecting cells. As the contact time becomes longer, the merit of no-culture detection decreased practically. Therefore, the contact time should be $2 \mathrm{hr}$. The cell number per colony of $L$. brevis cultivated on M-NBB medium for $24 \mathrm{hr}$ were 20-400 (Takahashi et al., 2000b); thus, the doubling time of $L$. brevis was inferred 2.5-5 h. At $2 \mathrm{hr}$ of incubation, $L$. brevis was considered to be in a single cell state.

\section{Detection accuracy test}

The cell suspension of $L$. brevis SBC 8001, with varying mixed ratio of viable and non-viable cells, was measured with RMDS and C.P.C. method. Fig. 7 shows the relationship between the viability and detection count with RMDS and C.P.C method. Detection ratio with RMDS and C.P.C. method showed a 1:1 correlation to the viability, and each method could detect only viable cells. In case of $0 \%$ viability, both methods did not detect any cells, indicating that no false positive results were obtained. The correlation of the count of detection between two methods in this experiment was $R^{2}=0.8202, y=0.8942 x$ when $x$ was RMDS and $y$ was 

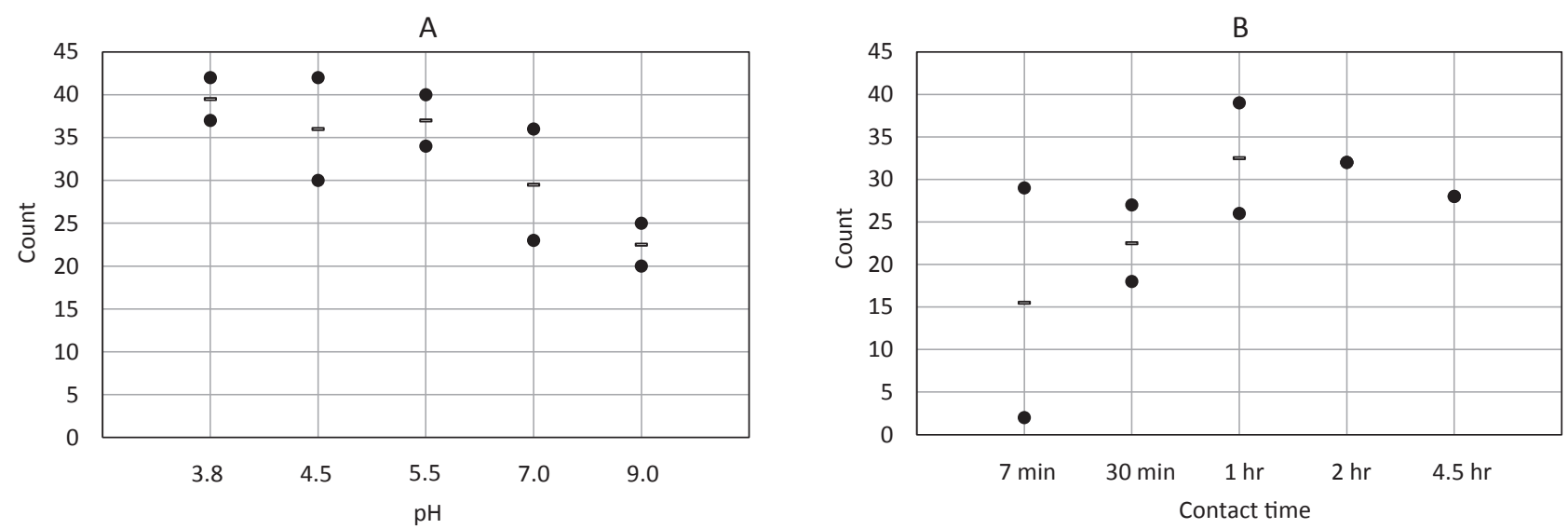

FIG. 6. (A) Influence of $\mathrm{pH}$ of the agar plate on detecting cells. Agar plates containing $1 \%$ glucose, $0.2 \%$ malic acid, and $0.67 \%$ YNB were adjusted to $\mathrm{pH} 3.8,4.5,5.5,7.0$, and 9.0. Precipitation of components was observed at pH 10.0. L. brevis SBC 8001 cultivated in MRS medium was used for this test $(n=2)$.

(B) Effect of the incubation time of the filter on the agar plate for detecting cells $(n=2)$. Agar plates ( $1 \%$ glucose, $0.2 \%$ malic acid, and 0.67\% YNB; pH 5.2) and L. brevis SBC 8001 cultivated in MRS medium were used for this test $(n=2)$. Symbols; ; measured value, $\boldsymbol{-}$; mean value.
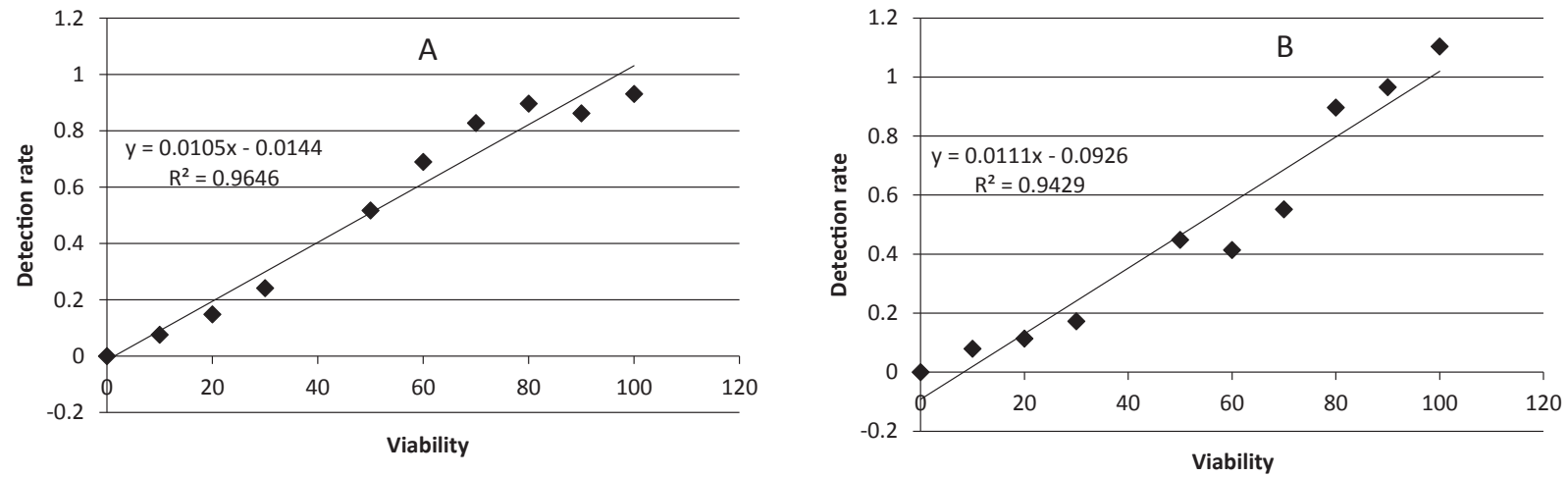

FIG. 7. Relationship between viability and detection count (A, RMDS method; B, C.P.C. method). L. brevis SBC 8001 cultured in MRS medium was used. The definition of viability is described in MATERIALS and METHODS. Detection rate was calculated as mentioned below: (Detection rate) $=$ (Count of RMDS or C. P. C method)/(Number of added cells; 29) $\times 100$.

C.P.C. and it was considered that there was no difference in the detection results.

When the cell suspension (L. brevis SBC 8002 cultivated in beer) was filtered by adding approximately 20-30 cells to $350 \mathrm{ml}$ of beer, the detection count of RMDS was 18, 13, 39 and that for C.P.C. method was $15,9,14 \mathrm{CFU}$ (96 hr of cultivation on M-NBB medium at $30^{\circ} \mathrm{C}$ ) (data not shown). There was no statistically significant difference in the data of both methods and it was considered that there was no difference in the detection results.

We decreased the external noise by removing the ATP derived from the beer remaining on the filter that impeded the detection of ATP contained in lactic acid bacterial cells (Fig. 8). The sample beer is filtered as shown in MATERIALS AND METHODS "Beer filtration", the membrane filter is contacted with GMY agar plate at room temperature for $2 \mathrm{hr}$ and measured with RMDS. This method can measure approximately 1-100 viable lactic acid bacterial cells, which contaminating a few to several liters of beer, without the need for cultivation. We developed a simple and practical method that can detect less than 10 lactic acid bacterial cells contaminating the beer of which contain a high amount of ATP in approximately $2 \mathrm{hr}$ without culturing. It was possible to determine the contamination by lactic acid bacteria, which is a major problem in the quality control of beer immediately after production. We believe that this method can highly benefit stock inventory management and supply and demand by drastically decreasing the 
Pouring the beer (350-633 $\mathrm{ml}$ ) into the funnel Stand until a part of the liquid level could be visually confirmed

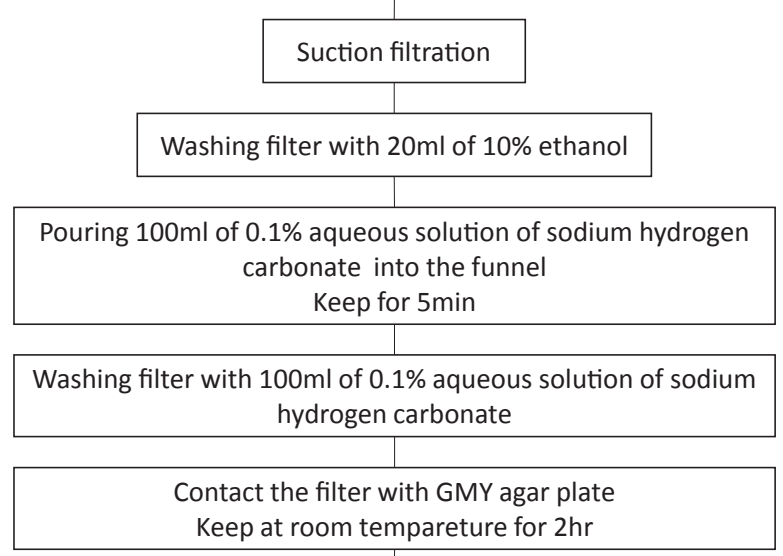

Measurement with RMDS

FIG. 8. Method of single lactic acid bacterial cell detection in beer by RMDS.

time needed for testing for microbial contamination.

\section{REFERENCES}

Boyaval P. (1989) Lactic acid bacteria and metal ions. Lait, 69(2), 87-113.

Hill A. E. (2015) Traditional methods of detection and identification of brewery spoilage organisms., P271-286. Brewing
Microbiology $1^{\text {st }}$ Edition, Woodhead Publishing.

Igimi S., Ezaki T., Takatori K., and Tsuchido T. (2013) Guidebook of Easy and Rapid Microbial Test Method (in Japanese), Technosystem, Tokyo.

Ogino K., and Hayashi H. (1977) Studies of the Builder Effects on Detergency, I. Sequestration Capacities of Some Organic Compounds. Jpn. Oil Chemistes' Society (in Japanese), 26 (5), 278-282.

Nishikawa N. and Kohgo M. (1996) Microbial control in the brewery. MBAA Tech. Quart., 22, 61-66.

Odaka H., Fuuda K., Mizuochi S., and Horigome K. (1996) Adenosine Triphosphate Content of Microorganisms Related with Food Spoilage. Jpn. J. Food Microbiol., 13, 29-34.

Priest, F. G. (1995) Gram-positive brewery bacteria., P.127161. In Priest, F. G. and Campbell, I. (ed.), Brewing microbiology. Chapman \& Hall, London.

Sakakibara T., and Imai K. (2003) Enumeration of bacterial cell numbers by amplified firefly bioluminescence without cultivation. Anal. Biochem., 312, 48-56.

Sato T. and Nakakita Y., Japan Kokai Tokkyo Koho, 07-053757 (Feb. 20, 1995).

Takahashi T., Nakakita Y., Nara Y., Uehara A., Monji Y., Watari J., and Shinotsuka K. (1999) Application of automatic Micro-Star-RMDS-SPS (ATP bioluminescence method) to product testing in brewery. Bokin Bobai (in Japanese), 27, 759-764.

Takahashi T., Nakakita Y., Watari J., and Shinotsuka K. (2000a) Application of bioluminescence method for the beer industry: Sensitivity of MicroStar ${ }^{T M}$-RMDS for detecting beer-spoilage bacteria. Biosci. Biotechnol. Biochem., 64(5), 1032-1037.

Takahashi T., Nakakita Y., Watari J., and Shinotsuka K. (2000b) A new rapid technique for detection of microorganisms using bioluminescence and fluorescence microscope method. J. Biosci. Bioeng., 89 (5), 509-513. 